\title{
Excitation of plasma waves by unstable photoelectron and thermal electron populations on closed magnetic field lines in the Martian ionosphere
}

\author{
N. Borisov ${ }^{1,2}$ and E. Nielsen ${ }^{2}$ \\ ${ }^{1}$ Institute of Terrestrial Magnetism, Ionosphere and Radio Waves propagation, 142190, Troitsk, Russia \\ ${ }^{2}$ Max-Planck Institut für Sonnensystemforschung, D-37191 Katlenburg-Lindau, Germany
}

Received: 2 November 2004 - Revised: 10 March 2005 - Accepted: 24 March 2005 - Published: 3 June 2005

\begin{abstract}
It is argued that anisotropic electron pitch angle distributions in the closed magnetic field regions of the Martian ionosphere gives rise to excitation of plasma instabilities. We discuss two types of instabilities that are excited by two different populations of electrons. First, the generation of Langmuir waves by photoelectrons with energies of the order of $10 \mathrm{eV}$ is investigated. It is predicted that the measured anisotropy of their pitch angle distribution at the heights $z \approx 400 \mathrm{~km}$ causes excitation of waves with frequencies $f \sim 30 \mathrm{kHz}$ and wavelengths $\lambda \sim 30 \mathrm{~m}$. Near the terminators the instability of the electrostatic waves with frequencies of the order of or less than the electron gyrofrequency exited by thermal electrons is predicted. The typical frequencies of these waves depend on the local magnitude of the magnetic field and can achieve values $f \sim 3-5 \mathrm{kHz}$ above strong crustal magnetic fields.
\end{abstract}

Keywords. Ionosphere (Planetary ionospheres; Plasma waves and instabilities) - Magnetospheric physics (Energetic particles)

\section{Introduction}

It is well-known that many different types of plasma instabilities are excited in planetary plasmas (Treumann, 2000). Radio waves are emitted in the plasma environment of planets whenever electrons are accelerated. In a plasma with a large number of free electrons accelerated in unison strong sources of wave radiation result. Waves may be excited owing to spatial anisotropies or spectral anomalies. The energy of the radiation may be taken from bulk flows or from energetic particle fluxes. The excited plasma waves propagate from the source region under control of the spatial variations in the background plasma and magnetic field. Space-borne plasma wave detectors have been used in the investigations of the complicated phenomena connected with plasma instabilities

Correspondence to: E. Nielsen

(nielsen@linmpi.mpg.de) in the Earth's ionosphere, especially in the auroral regions (Gurnett, 1974). Now significant attention is devoted to the exploration of the environment of Mars where an ionosphere similar to the Earth's ionosphere exists. In the present work we explore theoretically the possibility for wave activity in the closed magnetic field regions observed in the ionosphere of Mars.

On board the ESA spacecraft Mars Express the MARSIS experiment will probe the Martian plasma environment for plasma wave activity (Nielsen, 2004). These predictions of plasma wave activity in the Martian plasma environment are, therefore, to be experimentally tested in the near future.

Recently, it was discovered that strong local crustal magnetic fields exist on the surface of Mars, especially in the Southern Hemisphere (Acuña et al., 1998). These fields extend with significant amplitudes for hundreds of kilometers above the surface, forming mini-magnetospheres with closed magnetic field lines. At the footpoints the magnetic field can exceed large values of $H \approx 1000 \mathrm{nT}$. Even at the heights $z \sim 400 \mathrm{~km}$ these fields are strong enough to significantly to withstand the dynamical pressure of the solar wind.

Below the heights of $z \leq 200-250 \mathrm{~km}$ collisions between electrons and neutral particles are frequent, while above such heights the plasma can be considered as almost collisionless. Thus, electrons moving along a magnetic loop suffer collisions at low altuitudes while they are nearly collisionless near the peak of the loop. Regions of magnetic loops are surrounded by cusp-like regions of magnetic field lines which extend into the solar wind. The properties of the ionospheric plasma within closed magnetic flux tubes, therefore, deviate significantly from the surrounding plasma. According to Krymskii et al. (2003), in the closed tubes neutral particles are cooler and electrons are hotter than outside. One may speculate that electons are heated by some source inside magnetic loops (e.g. interaction with waves). As the exchange of energy across the magnetic field lines is reduced significantly in strong magnetic fields electrons are hotter in the loop than outside. Photoelectrons created and trapped on such crustal magnetic field loops were detected by the 


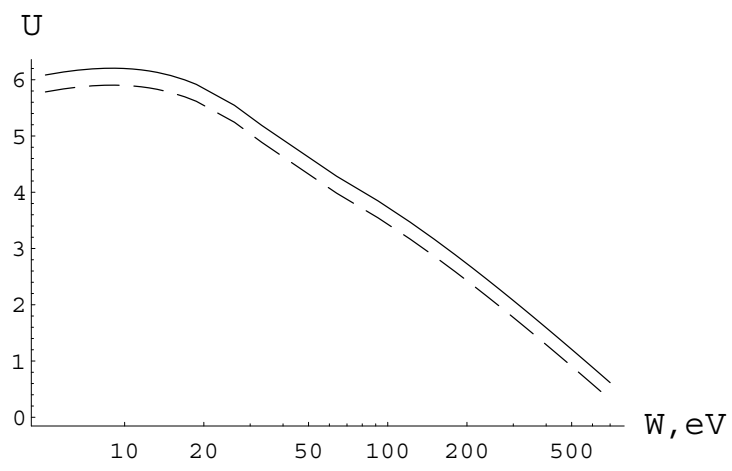

Fig. 1. Source-cone distribution function of photoelectron fluxes on closed magnetic field lines in the Martian ionosphere. Shown are the differential directional fluxes averaged over pitch angle (solid curve) and transverse fluxes (dashed) as functions of electron energy. The curves are based on Eqs. (7) and (13). Both axes are in logarithmic scale.

electron reflectometer (ER) on board Mars Global Surveyor (Mitchell et al., 2003). The distribution function of these hot electrons were investigated by Liemohn et al. (2003) and it was shown that it is characterized by a pitch angle anisotropy.

An anisotropy in the pitch angle distribution of charged particles leads to various plasma instabilities (Mikhailovskii, 1974). The aim of this work is to attract attention to the possibility of wave excitation on closed magnetic field lines in the ionosphere of Mars. Two types of instabilities are theoretically investigated. These instabilities are associated with two distinctly different electron pitch angle distributions and electron energy ranges. We show that the observed sourcecone pitch angle distribution of photoelectrons with minimum flux at 90 deg pitch angle (Liemohn et al., 2003) can be the source of Langmuir waves. According to our analysis the instability is strong enough that the excited waves might be detected by the MARSIS wave detector. Then we consider the excitation of electrostatic waves with the frequencies of the order of the electron cyclotron frequency. The source of this instability is the anisotropic loss-cone distribution (maximum flux at $90 \mathrm{deg}$ pitch angle) of thermal electrons. We argue that such a type of anisotropy should be more pronounced in the vicinity of the terminator, where the population of transit electrons (i.e. electrons with small pitch angle) quickly decreases in the absence of solar UV radiation. The two different electron populations are presented in the next two sections, and finally summerized and discussed.

\section{Unstable photoelectron population}

2.1 Conceptual model of photoelectron distribution on closed magnetic field lines

Recently, observations of the pitch angle distribution and energy spectra of photoelectrons in the Martian ionosphere were discussed by Liemohn et al. (2003). The observations were made in regions of closed magnetic fields, i.e. the measured electron fluxes were on magnetic loops with both ends of the magnetic field lines entering the neutral atmosphere. It was found that the pitch angle distribution of electrons with the energies from $10 \mathrm{eV}$ to $300 \mathrm{eV}$ was characterized by a minimum at $90 \mathrm{deg}$, while at higher energies, up to $700 \mathrm{eV}$, the distribution became nearly isotropic. This latter observation was suggested to indicate increased efficiency of scattering with growing energy, with the effect of filling in the minimum at $90 \mathrm{deg}$ at high energies. The energy spectrum of the fluxes averaged over pitch angle had a power law dependence. In the case of equilibrium a spectrum is typically Maxwellian. The power law dependence indicates a lack of equilibrium, suggesting that the collision frequency between electrons is too small to support the total thermal equilibrium.

These observations are indicative of the following scenario. Photoelectrons are generated by solar UV radiation interacting by scattering with the neutral atmosphere at low altitudes. This interaction results in a flow of photoelectrons from the top of the neutral atmosphere into the upward hemisphere. These electrons now propagate along the magnetic field lines conserving their magnetic momentum, such that a concentration of electrons with increased parallel velocities to the magnetic field will occur. This process will take place for electrons originating in both hemispheres (north and south). As a result, a pitch angle distribution near the top of the magnetic field lines with a minimum at $90 \mathrm{deg}$ is produced. Such a pitch angle distribution is referred to as a source-cone distribution. While there is strong enough scattering of electrons at low altitudes, at the top of the magnetic loops, according to our estimates, the scattering is weak. This prevents the $90 \mathrm{deg}$ minimum from filling-in, and also accounts for the observed power law energy spectrum. In this work it is shown that the pitch angle anisotropy of low energy photoelectrons $(W \leq 10 \mathrm{eV})$ at the top of closed magnetic field lines can lead to excitation of Langmuir plasma waves.

In the following section an analytical model of electron distribution functions is intoduced to allow a quantitative description of the energy spectra and pitch angle distribution. Figure 1 shows a typical pitch angle averaged (solid) and the transverse (dashed) electron fluxes versus energy associated with the model distribution function. The presented curves qualitatively reproduce the real pitch angle anisotropy of photoelectrons measured inside magnetic loops and their energy distribution. More detailed analysis of the problem will be given in the next section. This is followed by a discussion of excitation of Langmuir waves, including numerical estimates.

\subsection{Distribution of photoelectrons in crustal magnetic loops}

Suppose a magnetic loop is symmetric with respect to the point where the magnetic field is horizontal. With "s" being the coordinate along the field line let the coordinate of the horizontal point be $s=0$ while the two feet (the boundaries 
where plasma is still collisionless) have coordinates $s= \pm L$. As photoelectrons are created at both ends of the magnetic field line and travel upward their distribution is presented as

$F_{p h}=F_{p h}^{(+)}+F_{p h}^{(-)}$,

where $F_{p h}^{( \pm)}$is the distribution of photoelectrons penetrating into the upper part of the loop from the two ends of the field line. The distribution $F_{p h}^{(+)}$contains electrons with positive longitudinal velocity $v_{\|}>0$ while $F_{p h}^{(-)}$describes electrons with negative longitudinal velocities. The distribution functions $F_{p h}^{( \pm)}$are found as the stationary solutions of the Boltzmann kinetic equation

$\frac{\partial F_{p h}^{( \pm)}}{\partial t}+\mathbf{v} \nabla F_{p h}^{( \pm)}-\frac{e}{m} \mathbf{E} \frac{\partial F_{p h}^{( \pm)}}{\partial \mathbf{v}}-\omega_{H e}[\mathbf{v} \times \mathbf{h}] \frac{\partial F_{(p h)}^{ \pm}}{\partial \mathbf{v}}=0$

with the boundary conditions

$F_{p h}^{(+)}(-L)=F_{b}^{(+)}(\mathbf{v}, \mu), F_{p h}^{(-)}(L)=F_{b}^{(-)}(\mathbf{v}, \mu)$.

Here $\mathbf{v},-e, m$ are the velocity, the charge and the mass of electron, $\mathbf{E}$ is electric field, $\omega_{H e}=e H / m c$ is the gyrofrequency of elecrons, $\boldsymbol{H}$ is the magnetic field, c is the speed of light, $\mathbf{h}$ is a unit vector along the magnetic field line, $\mu=\cos \theta, \theta$ is a pitch angle.

Taking into account that the magnetic field changes significantly along the field line we rewrite Eq. (2) for the stationary distribution of electrons in the upper (collisionless) part of the loop in the form (Lejeune and Wormser, 1976)

$v \mu \frac{\partial F_{p h}^{( \pm)}}{\partial s}-v \frac{1-\mu^{2}}{2 H(s)} \frac{d H}{d s} \frac{\partial F_{p h}^{( \pm)}}{\partial \mu}=0$.

Here $H(s)$ is the absolute value of the magnetic field. The stationary electric field is assumed to be so weak that it does not influence the motion of photoelectrons and therefore it has been neglected in Eq. (4).

The functions $F_{p h}^{( \pm)}(\mp L)=F_{b}^{( \pm)}(\boldsymbol{v}, \mu)$ describing the distribution of photoelectrons at the ends of the crustal loops in the Mars' ionosphere are not known. At the same time there is an indication based on the experimental data that the pitch angle distribution of photoelectrons in the upper part of the loop is anisotropic (Liemohn et al., 2003). It is seen from Fig. 2 (in Liemohn et al., 2003) that in some cases the parallel flux of photoelectrons is larger than the flux in the transverse plane. Such an anisotropic distribution is called a source-cone distribution.

Let us now discuss possible analytical approximations for the distribution of photoelectrons in the upper part of crustal magnetic tubes. Taking into accout that the pitch angle averaged flux is observed to quickly decrease with increasing energy (as presented in Fig. 3, Liemohn et al. (2003)) we expect that the differential flux should have a power law spectrum at high energies or even to contain an exponential component (Maxwellian) with some effective energy $W_{p h}$ at low

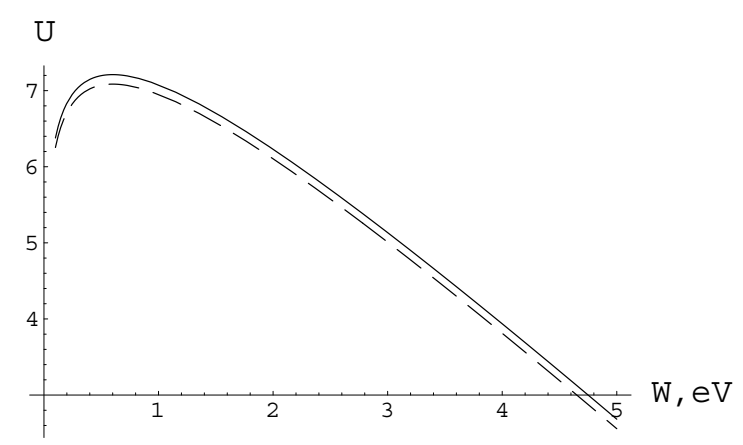

Fig. 2. Loss-cone distribution function of thermal electrons on closed magnetic field lines in the Martian ionosphere. Shown are the differential directional fluxes averaged over pitch angle (dashed curve) and transverse fluxes (solid) as a function of electron energy. The curves are based on Eq. (26). Both axes are in logarithmic scale.

energies. It is possible to find several analytical solutions of Eq. (2) with such a component. The most simple of them is the Maxwellian distribution function:

$F_{1}=\frac{A_{1}}{\left(\sqrt{2} v_{p h}\right)^{3}} \exp \left[-\frac{v^{2}}{2 v_{p h}^{2}}\right]$.

The flux of electrons described by this function is isotropic in pitch angles. Hence, it does not correspond to the existing data. Another function is the so-called loss-cone distribution,

$F_{2}=A_{2} \frac{H^{j}(0)\left(v^{2}\left(1-\mu^{2}\right)\right)^{j}}{H^{j}(s)\left(\sqrt{2} v_{p h}\right)^{2 j+3}} \exp \left[-\frac{v^{2}}{2 v_{p h}^{2}}\right], \quad j=1,2, .$.

It describes the electrons trapped in a magnetic "bottle". The peculiarity of this distribution is the lack of electrons with large longitudinal velocities. Hence the corresponding flux has maximum at pitch angles close to $\theta=90^{\circ}$ instead of a minimum . At last we present the function in which the amount of electrons with large longitudinal velocities is enhanced:

$F_{3}=A_{3} \frac{\left(v^{2}\left(1-\left(1-\mu^{2}\right) \frac{H(0))}{H(s)}\right)^{j}\right.}{\left(\sqrt{2} v_{p h}\right)^{2 j+3}} F\left(v^{2}\right), \quad j=1,2, \ldots$,

where $F\left(v^{2}\right)$ is a decreasing function of energy. For small energies $W \sim 10 \mathrm{eV}$ we take it in the form of the Maxwellian distribution Eq. (5). We consider Eq. (7) as a model distribution function of photoelectrons in the crustal loops. It is more convenient to use invariant variables $v, \xi$, instead of $v, \mu$, where

$\xi= \pm \sqrt{\left(1-\mu^{2}\right) \frac{H(0)}{H(s)}}$.

It is clear that $\xi$ changes within the interval $-\sqrt{\frac{H(0)}{H(L)}} \leq \xi \leq \sqrt{\frac{H(0)}{H(L)}}$. The longitudinal and the transverse velocities in coordinates $v, \xi$ take the form:

$v_{\|}= \pm v \sqrt{1-\frac{H(s)}{H(0)}} \xi^{2}, v_{\perp}=v \sqrt{\frac{H(s)}{H(0)}}|\xi|$ 

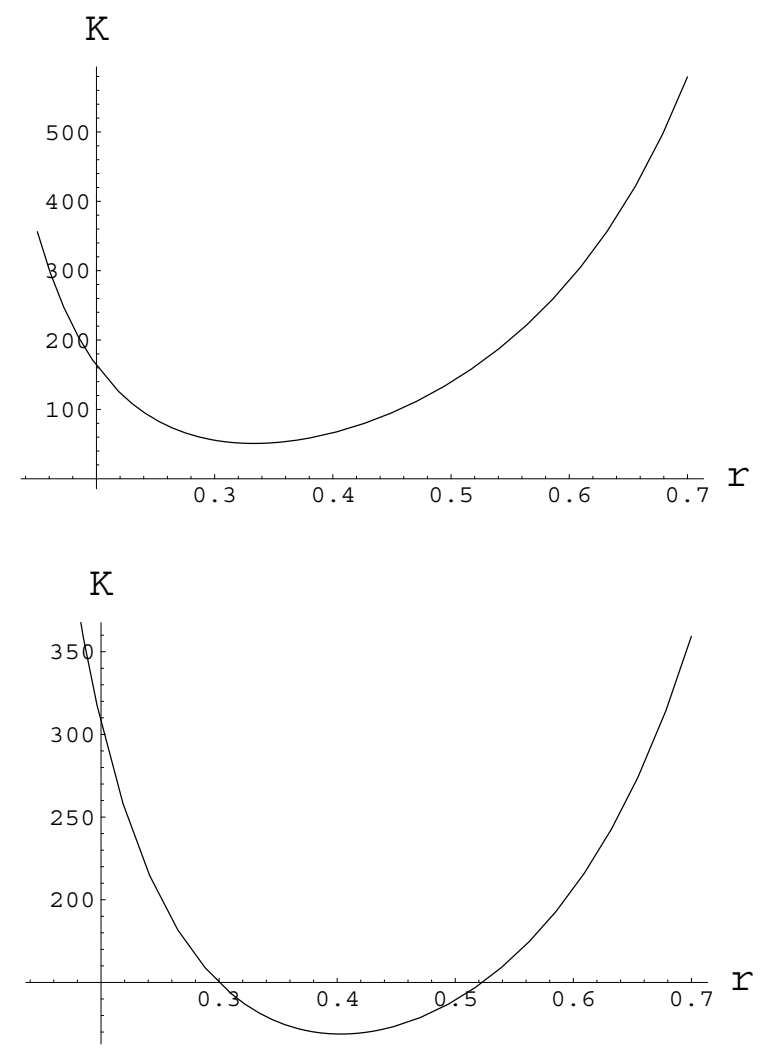

Fig. 3. (a) The real part (K) of the dispersion relation for thermal electron on closed magnetic field lines (Eq. (28)) at Mars shown versus frequency below the electron gyrofrequency, $r=\omega / \omega_{H e}$. This curve is for a ratio of 0.25 between the concentrations of the transient component and of the loss-cone distribution (the electron flux trapped in the "magnetic bottle"). The real part is positive in the whole frequency interval, indicating transparency. (b) Same as Fig. 3a, but here for a ratio of 0.4 between the concentration of the transient component and the component trapped in the "magnetic bottle". The real part is negative for $0.3<r<0.5$, indicating that waves within that range can not propagate. Thus, for increasing concentration in the magnetic bottle, (decreasing ration) the whole frequency range below the electron gyrofrequency becomes transparent (Fig. 3a).

Note that at the foot of a strong magnetic loop, where $H(0) / H(s) \ll 1$, the distribution presented in Eq. (7) is almost isotropic. At the same time, near the top of the loop, the pitch angle anisotropy is clearly seen. Now we are in a position to calculate with the help of Eq. (7) the pitch angle anisotropy of electron fluxes. Let us designate $\frac{d J_{\|}}{d W}$ and $\frac{d J_{\perp}}{d W}$ the differential fluxes of electrons that correspond to pitch-angle $\theta=0$ and $\theta=90^{\circ}$, correspondingly. The ratio of such fluxes depends on the parameter $j$ which is unknown. Taking for example $j=1$, we find for this ratio in the equatorial part $(s=0)$ and at the bottom of the loop $(s= \pm L)$ :

$\left.\frac{d J_{\|}}{d J_{\perp}}\right|_{s=0} \approx 0.75 \sqrt{\frac{H(L)}{H(0)}},\left.\frac{d J_{\|}}{d J_{\perp}}\right|_{s=L} \approx 0.8$.
Note that in the central part of the loop the parallel flux exceeds the transverse one several times because $H(L) \gg H(0)$, while at the bottom of the loop the two fluxes are close to each other. Also, it should be mentioned that the ratio of the fluxes according to Eq. (10) does not depend on energy.

To calculate the differential flux versus energy we need to specify the function $F\left(v^{2}\right)$ in Eq. (7). It should be chosen in such a way that the pitch angle averaged flux $d<J>/ d W$ reproduces the real dependence on energy measured by Liemohn et al. (2003). With the help of Eq. (7) such flux can be presented in the form:

$\frac{d<J>}{d W} \approx 5.5 \sqrt{\frac{H(0)}{H(L)}} \frac{A_{3}}{v_{p h}^{5}} W^{2} F(W)$,

where $W$ is the energy $W=m v^{2} / 2$ and $A_{3}$ is determined by the concentration of photoelectrons:

$N_{p h} \approx 1.1 \frac{A_{3}}{v_{p h}^{5}} \int v^{4} F\left(v^{2}\right) d v \int \frac{\xi^{2}}{\sqrt{1-\frac{H(s)}{H(0)} \xi^{2}}} d \xi$.

We suggest the following approximation for $F(W)$

$F(W)=\exp \left(-\frac{W}{W_{p h}^{(1)}}\right)+q\left(\frac{W_{p h}^{(2)}}{W+W_{p h}^{(2)}}\right)^{\alpha+j}$,

where $W_{p h}^{(1)}, W_{p h}^{(2)}$ are two characteristic energies, $W_{p h}^{(2)}>W_{p h}^{(l)}, q$ and $\alpha$ are two more unknown parameters which should be chosen to fit the experimental data. For small energies $W \sim W_{p h}^{(l)}$ the function $F(W)$ decreases exponentially $F(W) \approx \exp \left(-\frac{W}{W_{p h}^{(l)}}\right)$, while for large energies $W>W_{p h}^{(2)}$ it decreases according to power law $F(W) \sim\left(W_{p h}^{(2)} / W\right)^{\alpha+j}$. Taking into account the behaviour of the real pitch angle averaged electron flux presented by Liemohn et al. (2003) (see their Fig. 3) we are able to estimate the main parameters of the distribution function

$$
\begin{gathered}
N_{p h} \approx 2.8 A_{3} \approx 1.6 \mathrm{~cm}^{-3} W_{p h}^{(1)} \approx 4.4 \mathrm{eV}, \\
W_{p h}^{(2)} \approx 25 \mathrm{eV}, q \approx 0.1, \alpha \approx 5.3
\end{gathered}
$$

Two fluxes (pitch angle averaged and transverse) for this chosen distribution function are presented in Fig. 1.

\subsection{Excitation of Langmuir waves}

High frequency electrostatic waves are described by the Poisson equation:

$\Delta \phi=4 \pi e n$.

Here $\phi$ is the potential of the electric field $\boldsymbol{E}=-\nabla \phi, n$ is the perturbation of plasma concentration. The link between the two unknown functions $n$ and $\phi$ is given by the Boltzmann 
equation (Eq. (2)) for the distribution function of electrons. The total distribution function is presented as

$F_{e}=F_{0}+f_{e}$,

where $F_{0}$ is the stationary distribution of electrons within the crustal magnetic loop and $f_{e}$ is a small perturbation. The main part of the stationary distribution $F_{0}$ is given by the distribution of thermal electrons $F_{t h}$ while a small additional contribution is produced by photolelectrons with the distribution function $F_{p h}$ (Eq. (1)).

Assuming that the perturbation of the electrons' distribution $f_{e}$ and the electric potential $\phi$ has small spacial scales (compared with the longitudinal variation of the magnetic field and undisturbed plasma concentration) we use the geometric optics approximation:

$$
\begin{array}{r}
f_{e}=\tilde{f}_{e}(v, s) \exp \left[-i\left(\omega t-k_{\|} s-\mathbf{k}_{\perp} \mathbf{r}_{\perp}\right)\right] \\
\phi=\tilde{\phi}(s) \exp \left[-i\left(\omega t-k_{\|} s-\mathbf{k}_{\perp} \mathbf{r}_{\perp}\right)\right] .
\end{array}
$$

Here $\tilde{f}_{e}, \tilde{\phi}$ are the amplitudes slowly varying with s, $\omega$ is the frequency of the excited waves, $k_{\|}, \boldsymbol{k}_{\perp}$ are the wave numbers along and across the magnetic field. The substitution of Eq. (16) into Eq. (2) allows us to find the perturbation $\tilde{f}_{e}$

$$
-i\left(\omega-k_{\|} v_{\|}\right) \tilde{f}_{e}+\omega_{H e}[\mathbf{v} \times \mathbf{h}] \frac{\partial \tilde{f}_{e}}{\partial \mathbf{v}}=-i \frac{e}{m} k_{\|} \tilde{\phi} \frac{\partial F_{0}}{\partial v_{\|}},
$$

where $v_{\|}$is the longitudinal velocity of electrons. For the frequencies much higher than the Larmor frequency we can neglect the term containing the magnetic field. In this case it follows from Eq. (18) that

$\tilde{f}_{e}=\frac{e}{m} \frac{k_{\|} \tilde{\phi}}{\omega-k_{\|} v_{\|}} \frac{\partial F_{0}}{\partial v_{\|}}$.

Thermal electrons that give the main input to plasma perturbation have rather small characteristic (thermal) velocity $v_{T e} \ll \omega / k_{\|}$. This is why they do not contribute to the resonance $\omega=k_{\|} v_{\|}$, (the pole in Eq. (19)). As a result we arrive at the following approximate expression for the perturbed plasma concentration:

$\tilde{n}=-\frac{e}{m} \frac{k_{\|}^{2} \tilde{\phi}}{\omega^{2}} \int\left(1+3 \frac{k_{\|}^{2} v_{\|}^{2}}{\omega^{2}}\right) F_{t h}(v) d^{3} v$.

The substitution of Eq. (20) into the Poisson equation allows us to find the frequency of perturbations

$\omega^{2}=\frac{4 \pi e^{2}}{m} \int\left(1+3 \frac{k_{\|}^{2} v_{\|}^{2}}{\omega^{2}}\right) F_{t h}(v) d^{3} v$.

The dispersion relation Eq. (21) describes Langmuir waves with thermal corrections $\propto k_{\|}^{2} v_{T e}^{2} / \omega^{2}$. Note that $\int F_{t h} d^{3} v$ is equal to $N_{t h}-$ the concentration of thermal electrons. In deriving Eq. (21) we have neglected the contribution of photoelectrons. Such electrons have large velocities and due to this they determine the input of the resonance $\omega=k_{\|} v_{\|}$to the dispersion relation. This resonance corresponds to the well-known Landau damping, if the derivative $\partial F_{p h} / \partial v_{\|}$is negative at the resonance point $v_{r}=\omega / k_{\|}$and describes the growth rate if the derivative is positive. Taking into account the above mentioned resonance we find the perturbation of the plasma concentration has the form (compare to Eq. (21))

$$
\begin{aligned}
\tilde{n}= & -\frac{e}{m} \frac{k_{\|}^{2} \tilde{\phi}}{\omega^{2}} \int\left(1+3 \frac{k_{\|}^{2} v_{\|}^{2}}{\omega^{2}}\right) F_{t h}(v) d^{3} v \\
& -i \frac{e}{m} \frac{k_{\|} \tilde{\phi}}{\omega} \int \frac{\partial F_{p h}}{\partial v_{\|}} \delta\left(\omega-k_{\|} v_{\|}\right) d^{3} v .
\end{aligned}
$$

It is seen from Eq. (22) that positive values of the derivative $\partial F_{p h} / \partial v_{\|}$give rise to plasma instability with the growth rate $\gamma$

$\left.\gamma \approx \frac{4 \pi e^{2}}{m} \frac{\omega_{P e}\left(N_{t h}\right)}{k_{\|}^{2}} \int \frac{\partial F_{p h}}{\partial v_{\|}}\right|_{v_{r}} d^{2} v_{\perp}$.

It is clear that the peculiarities of the photoelectrons' distribution function in the magnetic tube influences the growth rate of the instability.

\subsection{Typical parameters of the excited waves}

Let us now find the derivative $\frac{\partial F_{p h}}{\partial v_{\|}}$that enters the growth rate of instability. Note that the distribution of photoelectrons in the upper part of the loop consists of two parts $F_{p h}^{( \pm)}$(see Eq. (1)). Suppose for certainty that we investigate the excitation of the Langmuir waves with $\omega>0, k_{\|}>0$. The resonance velocity for these waves is also positive $v_{r}>0$. It means that only one part $\left(F_{p h}^{(+)}\right)$of the distribution contains photoelectrons that can be in resonance with such waves. For the waves propagating in the opposite direction (negative resonance velocity), another part $\left(F_{p h}^{(-)}\right)$in the distribution should be taken into account. Since we are interested in electrons with the energies $W \approx W_{p h}$, the part that contributes to the power law spectrum in Eq. (13) can be neglected. For the central part of the loop, where $H(s)=H(0)$, the required derivative $\frac{\partial F_{p h}}{\partial v_{\|}}$ takes the form

$$
\frac{\partial F_{p h}}{\partial v_{\|}}=0.4 N_{p h}\left(1-\frac{v_{\|}^{2}}{2 v_{p h}^{2}}\right) \frac{2 v_{\|}}{\left(\sqrt{2} v_{p h}\right)^{5}} \exp \left[-\frac{v^{2}}{2 v_{p h}^{2}}\right] .
$$

It is seen that the derivative is positive for small parallel velocities $v_{\|}<\sqrt{2} v_{p h}$. At the same time, according to Eq. (7), the amount of photoelectrons decreases with $v_{\|} \rightarrow 0$. It means that the maximal growth rate is expected for resonance velocities close to $v_{p h}$. The integration with respect to $v_{\perp}$ in Eq. (23) is easily fulfilled. As a result we arrive at the final expression for the growth rate of the instability:

$$
\gamma=\frac{\omega_{P e}\left(N_{t h}\right) \omega_{P e}^{2}\left(N_{p h}\right)}{\sqrt{2 \pi} k_{\|}^{2} v_{p h}^{2}} \frac{v_{r}}{\sqrt{2} v_{p h}}\left(1-\frac{v_{r}^{2}}{2 v_{p h}^{2}}\right) \exp \left[-\frac{v_{r}^{2}}{2 v_{p h}^{2}}\right] .
$$


It can be proved that the maximal growth rate is achieved at $v_{r} \approx 1.2 v_{p h}$. After the substitution in Eq. (25) the main parameters $N_{t h}=20 \mathrm{~cm}^{-3}, N_{p h}=1.6 \mathrm{~cm}^{-3}, v_{p h}=9.10^{7} \mathrm{~cm} / \mathrm{s}$ we estimate the maximal growth rate of the instability as $\gamma_{\max } \approx 1,2.10^{3} \mathrm{~s}^{-1}$ and the characteristic wave number as $k_{\|}=\omega_{P e}\left(N_{t h}\right) / v_{r} \approx 2,4.10^{-3} \mathrm{~cm}^{-1}$. It is seen that the growth rate is large enough to provide significant amplitudes of the Langmuir waves.

\section{Unstable thermal electrons population}

3.1 Conceptual model of thermal electron distribution on closed magnetic field lines

Not only photoelectrons but also thermal electrons will spread along the closed magnetic field lines. Note that the concentration of thermal electrons is much larger than the concentration of photoelectrons. The scattering of thermal electrons due to their low energies is stronger than for photoelectrons. This is consistent with the general trend that the particle scattering cross section increases with decreasing energy for Coulomb collisions. Like the photoelectrons the thermal electrons stream along the magnetic field lines to form a source-cone distribution at high altitudes. Note in this scenario that the electrons are lost in the opposite hemisphere after one bounce. One may refer to the source-cone particles as "transient particles". However, owing to scattering, electrons are transferred (mainly) towards larger pitch angles such that some electrons become trapped. The trapped population will sum-up over time to produce a flux around $90 \mathrm{deg}$ pitch angle which will be equal to or less than the transient flux. However, near the terminator, for example, recombination at low altitudes may reduce the transient fluxes. As a result, the trapped fluxes may exceed the transient fluxes to form a loss-cone pitch angle distribution with maximum flux at $90 \mathrm{deg}$. Figure 2 shows the pitch angle averaged flux (dashed) and transverse flux (solid) of thermal electrons versus energy that corresponds to the loss-cone distribution. It will be shown that the pitch angle anisotropy of these low energy thermal electrons $(W<1 \mathrm{eV})$ can lead to excitation of plasma waves with frequencies of the order of or less than the electron cyclotron frequency.

The electrostatic waves with frequencies below the electron gyrofrequency is often called electrostatic whistlers. They are converted into electromagnetic whistler mode waves by the scattering on plasma irregularities. Such linear mechanism of wave transformation is well-known for different types of waves (see e.g. Erokhin and Moiseev, 1979). The observed isotropy of the energetic electron fluxes $(W>300 \mathrm{eV})$ can be explained by the interaction of such electrons with electromagnetic whistler mode waves. Indeed, such interaction is quite efficient because the longitudinal phase speed of waves is of the order of the characteristic velocity of fast electrons.
3.2 Excitation of waves with the frequencies of the order of electron cyclotron frequency

In the previous sections the instability excited by photoelectrons that have anisotropy with respect to their parallel velocity was investigated. In this case thermal electrons play a passive role and contribute only to the real part of the frequency. Now we discuss the instability excited by thermal electrons. Thermal electrons penetrate into the upper part of the loop from below where they have a Maxwellian distribution. But in the upper part of the loop another population of thermal electrons appears. Due to rare collisions some electrons become trapped in the inhomogeneous magnetic field (magnetic "bottle"). As a result their distribution takes the form of the loss-cone type (see Eq. (6)). Under stationary conditions the concentration of trapped electrons is smaller or of the order of the concentration of transit electrons. But near the terminator it is quite probable that the concentration of trapped electrons becomes larger than the transit ones. Indeed, without UV radiation electrons begin to disappear due to recombination, attachment, transverse drift, diffusion. In the lower part of the loop, where the concentration of ions is large, the recombination with $O_{2}^{+}$ions, according to our estimates, is a main mechanism of loss for transit electrons. Chemical reaction rates in the Mars' ionosphere can be found in Krasnopolsky (2002). As for the trapped electrons they oscillate in the upper part of the loop where the concentrations of neutrals and ions are small. The main mechanism of losses for them is the scattering into the loss-cone due to collisions. But it is known that the characteristic time of such scattering in the magnetic "bottle" is much larger than the inverse collision frequency of electrons (Pastukhov, 1987). So it is possible to expect that trapped electrons disappear more slowly than transit ones. As a result the distribution function of thermal electrons near the terminator can be modelled by the combination of the loss-cone distribution

$$
F_{j}^{(e)}\left(v_{\perp}, v_{\|}\right)=N_{B} \frac{v_{\perp}^{2 j}}{\pi^{3 / 2} j !\left(\sqrt{2} v_{t h}\right)^{2 j+3}} \exp \left[-\left(\frac{v_{\perp}^{2}+v_{\|}^{2}}{\sqrt{2} v_{t h}^{2}}\right)\right]
$$

and a small admixture of the Maxwellian distribution

$$
F_{M}=\frac{N_{M}}{\pi^{3 / 2}\left(\sqrt{2} v_{t h}\right)^{3}} \exp \left(-\frac{v^{2}}{2 v_{t h}^{2}}\right)
$$

where $N_{B}, N_{M}$ are the concentrations of the trapped electrons in the magnetic "bottle" and transit electrons, $j=1,2, \ldots$

The theory predicts that due to loss-cone distribution plasma becomes unstable (Mikhailovskii, 1974) and different types of waves (electomagnetic and electrostatic) can be excited. In the Earth's magnetosphere such distribution is responsible for the excitation of Alfvén waves and whistler mode waves in the radiation belts (Bespalov and Trakhtengerts, 1986) and also electrostatic waves with frequencies of the order of the electron cyclotron frequency in the equatorial part of the magnetosphere (Kennel et al., 1970). 
The procedure of obtaining the frequency and the growth rate of instability is the same as described in Sect. 2.3. First, we need to find the perturbation of the distribution function in the magnetic field. The corresponding solution of the Boltzmann equation, Eq. (2), as an infinite sum with respect to different gyroharmonics is well-known (see, e.g. Mikhailovskii, 1974). After the substitution of the concentration $n(\phi)$ into Eq. (1) we arrive at the dispersion relation. In the general case the real and the imaginary parts exist in this equation. Assuming that the imaginary part of the frequency (the growth rate) is small compared with the real one, we are able to split the dispersion relation into two different equations. It allows us to use the results presented by Karpman et al. (1974) with a small modification. Let us consider the most simple case when the transverse k-number of perturbations is large enough $k_{\perp}^{2} \rho_{e}^{2} \gg 1$ while the longitudinal k-number is small $a_{n}=\frac{\omega-n \omega_{H e}}{\left|k_{\|}\right| v_{\| e}} \gg 1$. Here $\rho_{e}=\frac{v_{T e}}{\omega_{H e}}$ is the Larmor radius of electrons., $n=0, \pm 1, \pm 2, \ldots$ In this case the real part of the dispersion relation reduces to

$$
\begin{aligned}
& \left(k_{\perp} \rho_{e}\right)^{3}+2 \frac{\omega_{P e}^{(M) 2}}{\omega_{H e}^{2}} k_{\perp} \rho_{e}=-\sqrt{\pi} \frac{\omega_{P e}^{(M) 2}}{\omega_{H e}^{2}} 2 \\
& {\left[\left(\frac{\alpha_{j}}{2 j-1}-\frac{N_{M}}{N_{B}}\right)\left(r \cot \pi r+\frac{\pi^{2} r \cot \pi r}{2 a^{2} \sin ^{2} \pi r}\right)-\frac{\alpha_{j}}{2 j-1} \frac{\pi U_{j}}{2 a^{2} \sin ^{2} \pi r}\right]}
\end{aligned}
$$

Here $r=\omega / \omega_{H e}$ is the reduced frequency, $\omega_{P e}^{(M)}, \omega_{P e}^{(B)}$ are the plasma frequencies that correspond to the concentrations $N_{M}, N_{B}$,

$U_{j}=2 j, \alpha_{j}=\frac{(2 j-1) ! !}{2^{j} j !}, a=\frac{\omega_{H e}}{\left|k_{\|}\right| v_{e}}$.

Further, we consider the case when the loss-cone is not too strong and set $j=1, U=2$. As a result, the imaginary part of the dispersion relation that determines the growth rate of instability takes the form:

$$
\begin{aligned}
g= & -\frac{a}{\sqrt{\pi} D} \sum_{n=-\infty}^{n=\infty}\left(U(r-n)-\left(1-\frac{N_{M}}{N_{B}}\right) r\right) \\
& \exp \left[-a^{2}(r-n)^{2}\right]-\frac{v_{e i}}{\omega_{H e}},
\end{aligned}
$$

where $g=\frac{\gamma}{\omega_{H}}$ is the reduced growth rate. Here $v_{e i}$ is the collision frequency of electrons with ions,

$$
\begin{aligned}
& D=\left(1-2 \frac{N_{M}}{N_{B}}\right)\left[\cot \pi r\left(1+\frac{\pi^{2}}{2 a^{2} \sin ^{2} \pi r}\right)\right. \\
& \left.-\frac{\pi r}{\sin ^{2} \pi r}\left(1+\frac{\pi^{2}}{2 a^{2}}\left(3 \cot ^{2} \pi r+1\right)\right)\right]+\frac{\pi^{2} U_{1}}{a^{2} \sin ^{2} \pi r} \cot \pi r .
\end{aligned}
$$

In the case if the Maxwellian component is absent $\left(N_{M}=0\right)$, the above obtained results coinCide with the results presented in Karpman et al. (1974). Note that the positive sign $g>0$ corresponds to the instability while the negative sign $g<0$ determines the attenuation of waves. In the discussed approximation we can also find the transverse $V_{g \perp}$ group velocity of the excited wave:

$V_{g \perp} \approx-v_{T e} \frac{3}{D \sqrt{\pi}}\left(\frac{\omega_{H e}}{\omega_{P e}^{(B)}}\right)^{2}\left(k_{\perp}^{2} \rho_{e}^{2}+2 \frac{\omega_{P e}^{(M) 2}}{\omega_{H e}^{2}}\right)$.

The presented above results allow us to estimate the excitation of the electrostatic waves with the frequencies of the order of electron gyrofrequency within the closed magnetic field lines in the ionosphere of Mars.

Two obvious requirements have to be fulfilled simultaneously for the excitation of waves. First, the right-hand side of Eq. (28) should be positive and large enough (as according to our assumption $k_{\perp} \rho_{e} \gg 1$ ). Second, the growth rate (see Eq. (29)), should also be positive. In this case the amplitude of the wave packet localized in some region within the closed magnetic field line starts to grow in time. At the same time the packet moves along and across the magnetic field line with the group velocities $V_{g \|}, V_{g \perp}$, correspondingly. This motion provides the linear mechanism of the amplification because the parameters of plasma and magnetic field change in space. The energy density of the wave packet with the central Fourier harmonic $k_{0}$ calculated by Karpman et al. (1975) can be presented in the form:

$$
I_{k_{0}}=\frac{r\left|E_{k_{0}}\right|^{2}}{8 \pi} D \text {. }
$$

The propagation of the wave packet in the geometric optics approximation is described by equation

$$
\begin{aligned}
& \frac{\partial}{\partial t} I_{k_{0}}+\left(\mathbf{V}_{g \perp} \nabla_{\perp}+\mathbf{V}_{g \|} \nabla_{\|}\right) I_{k_{0}}= \\
& \quad 2 g_{k_{0}} \omega_{H e} I_{k_{0}}+\sum_{k_{1}} P\left(k_{0}, k_{1}\right) I_{k_{0}-k_{1}} I_{k_{1}} .
\end{aligned}
$$

The last term in Eq. (33) corresponds to the nonlinear interaction between waves that contributes to the stabilization and the formation of the stationary spectrum of waves. Below only linear effects are taken into account. Let us introduce the typical scales $L_{\perp}$ and $L_{\|}$for the inhomogeneities of the medium across and along the magnetic field lines. Assuming that the inhomogeneity across the magnetic field line is stronger than along the field line, we are able to estimate the amplification $Q$ of the excited wave in the linear approximation:

$Q \approx 2 \frac{g_{k_{0}} L_{\perp} \omega_{H e}}{V_{g \perp}}$.

If $Q \gg 1$ we expect strong amplification of waves. The ratio $g_{k_{0}} / V_{g \perp}$ that enters Eq. (34) can be presented in the explicit form taking into account Eqs. (28) and (30). As a result, we find the amplification $Q$ as the following:

$$
Q=\frac{2}{3} \frac{\omega_{P e}^{2}\left(N_{B}\right) L_{\perp}}{v_{T e}} \frac{a \omega_{H e}}{k_{\perp}^{2} v_{T_{e}}^{2}+2 \omega_{P e}^{2}\left(N_{M}\right)}
$$



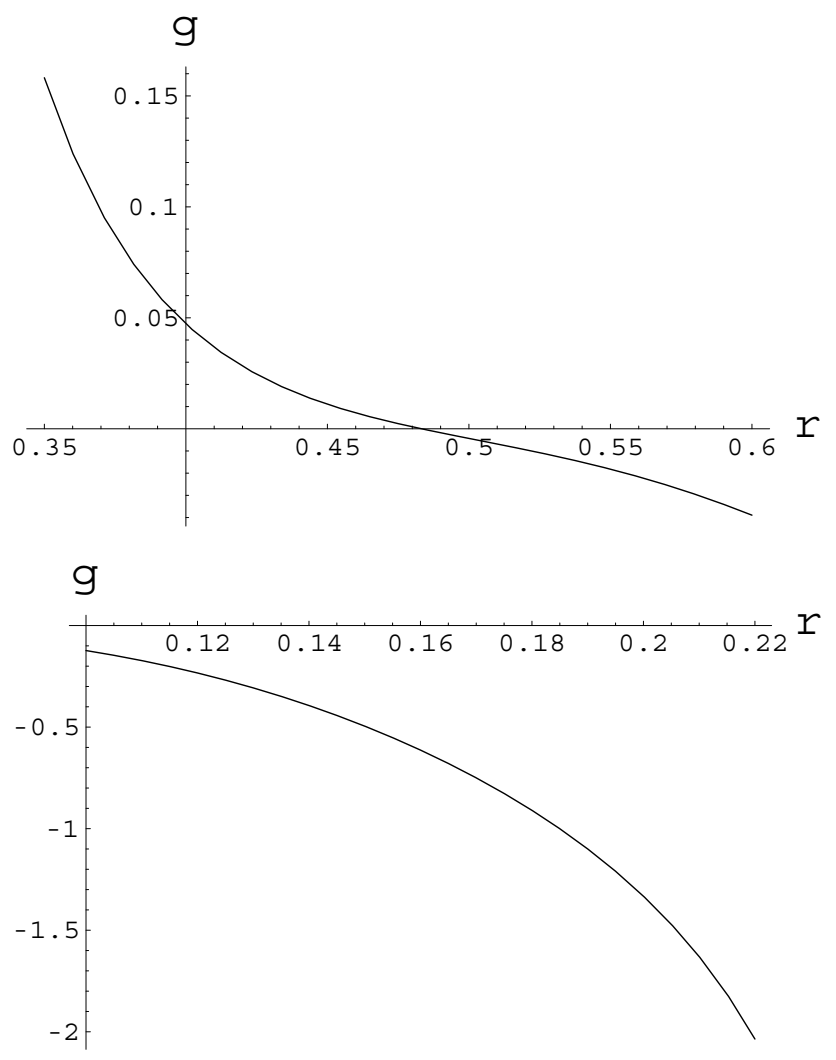

Fig. 4. (a) The imaginary part (g) of the dispersion relation of thermal electron on closed magnetic field lines (Eq. (29)) of Mars shown versus frequency. Both the frequency and imaginary part (or growth rate) are normalized to the electron gyrofrequency, $r=\omega / \omega_{H e}$. Only frequencies less than the gyrofrequency are considered. This curve is for a ratio of 0.25 between the concentration of the transient distribution and of the loss-cone distribution. For $r<0.47$ the growth rate is positive, indicting wave growth. (b) Same as Figure $4 \mathrm{a}$, but more for a ratio of 0.4 between the concentrations of the transient component and the trapped component. For this case the trapped component is reduced compared to the case shown in Fig. 4a. There is no growth of waves in the range $0.1<r<0.22$. Calculation also shows that for $r>0.56$ there is negative growth. For a ratio of 0.4 there are no unstable waves. Only for fairly large trapped thermal electron population (ratio $\sim 0.25$ ) are waves excited.

$$
\sum_{n=-\infty}^{n=\infty}\left[r\left(1+\frac{N_{M}}{N_{B}}\right)-2 n\right] \exp \left[-a^{2}(r-n)^{2}\right]
$$

Parameter $k_{\perp}^{2} v_{T_{e}}^{2}$ that enters the denominator of Eq. (35) can be found from Eq. (28). In the next section we present some numerical estimates for the amplification (35) on the closed magnetic field lines in the ionosphereof Mars.

\subsection{Numerical estimates for the waves excitation}

Our estimates are based on the available experimental data concerning the distribution of plasma concentration and magnetic fields in the ionosphere of Mars (see Acuña et al., 1998; Krymskii et al. 2003; Mitchell et al., 2001; Shinagawa, 2004). Unfortunately, these data are not sufficient for detailed analysis. This is why only rather crude estimates are given below. We use the data that correspond to the sunlight side and the height $z \approx 400 \mathrm{~km}$. For our calculations we need to specify several main parameters: $a, \omega_{P e}\left(N_{B}\right), N_{M} / N_{B}, v_{T e}$, $\omega_{H e}, v_{e i}$. Let us take $a=5, \omega_{P e} / \omega_{H e}=25, \omega_{H e}=10^{4} s^{-1}$, $v_{T e}=10^{7} \mathrm{~cm} / \mathrm{s}, v_{e i}=0.03 \mathrm{~s}^{-1}$. Our aim is twofold. First, to investigate how the ratio of concentrations $N_{M} / N_{B}$ influences the growth rate of the instability. It is clear that when this ratio becomes large enough the instability should disappear. Second, to find out at what frequency $r$ for a given ratio $N_{M} / N_{B}$ the strongest amplification is expected. In this paper we discuss only the frequency range $0<r<1$ where the largest growth rate for the given parameters is expected.

First, we calculate the right-hand side of Eq. (28) as a function of $r$ to find at what frequencies the plasma is transparent. In Figs. 3a and b the right-hand side of Eq. (28) is presented for the lowest frequency range $0<r<1$ for two ratios $N_{M} / N_{B}=0.25$ and $N_{M} / N_{B}=0.4$. It is seen that for a small reduced concentration of the Maxwellian plasma the whole region $0<r<1$ is transparent, while for a larger concentration $N_{M} / N_{B}=0.4$ there are no propagating waves in the region $0.3<r<0.52$.

The reduced growth rate $g=\gamma / \omega_{H e}$ as a function of $r$ for the same low range of frequencies and small ratio $N_{M} / N_{B}=0.25$ is presented in Fig. $4 \mathrm{a}$. We see that there is a restricted range of frequencies $r<0.47$ within which the positive growth rate exists. Note that a rather narrow region in the vicinity of $r \sim 0.28-0.33$ cannot be investigated in the chosen approximation. The denominator in Eq. (29) in this region tends to zero. The calculations of the growth rate for the second and larger ratio of concentrations is presented in Fig. $4 \mathrm{~b}$. It is clear from this figure that the instability dissapears. This result represents one of two regions where plasma is transparent $(r<0.3)$. For the other region, $r>0.56$, calculations also show that there is no instability. It means that the instability disappears for too little admixture of Maxwellian plasma (too large ratio $N_{M} / N_{B}$ ). The amplification factor Q calculated with the help of Eq. (34) $Q \sim 200$ shows that the waves with the reduced frequencies $r \sim 0.33-0.4$ should have large amplitudes. To estimate them correctly nonlinear processes should be taken into account. This problem will be investigated elsewhere.

Note that in the same manner the excitation of electrostatic waves with higher frequencies $\omega>\omega_{\mathrm{He}}$ can be investigated. It can be shown that the growth rate of the instability for such waves decreases to compare with the lowest frequency band $\omega<\omega_{H e}$. This is why we discussed only the excitation of waves with the frequencies below the gyrofrequency of electrons.

In our analysis we have discussed the case when the plasma frequency is much larger than the gyrofrequency of electrons. It allowed us to use rather simple formulas applicable for large transverse wave numbers $k_{\perp} \rho_{e} \gg 1$. We predict excitation of waves near the terminator where the plasma frequency is still not too small. Hence, we expect that the restriction $\omega_{P e} \gg \omega_{H e}$ is still valid near the terminator. During nighttime conditions (far from terminator), it is hardly 
possible to expect the excitation of waves. Therefore, we didn't discuss the case that corresponds to small plasma frequencies $\omega_{P e} \leq \omega_{H e}$.

\section{Discussion and conclusion}

We have suggested a model distribution function for photelectrons in the closed magnetic field regions, source-cone distribution, Eq. (7). It corresponds to the case when there are no collisions of photoelectrons in the upper part of the magnetic flux tubes. For such a distribution the flux of electrons along the field line is larger than the particle flux across the magnetic field. Since the instability is easier excited for an increased anisotropy, the analysis corresponds, therefore, to a conservative estimate. At the same time, for the chosen distribution this flux ratio does not depend on the energy (see Eq. (10)). According to Fig. 11 in Liemohn et al. (2003), the observed flux ratio fluctuates significantly with energy but, on average, it is almost constant, justifying the suggested distribution. With the help of this distribution function we have estimated the concentration $\left(N_{p h}=1.6 \mathrm{~cm}^{-3}\right)$ and the characteristic energy $\left(W_{r} \approx 4.4 \mathrm{eV}\right)$ of photoelectrons on the closed magnetic field lines. The introduced model pitch angle distribution of photoelectrons allows us to investigate the possible excitation of Langmuir waves and the growth rate of the instability. We expect the growth rate to be large enough for significant amplification of the Langmuir waves. The typical parameters of the excited waves are the following. At the height $z \approx 400 \mathrm{~km}$ on the sunlit side the frequency is of the order of $\omega_{P e} \approx 2.10^{5} \mathrm{~s}^{-1}$. It becomes larger on the inner magnetic field lines where the ionosphere is more dense. At the same time the range of the characteristic wavelengths of the excited perturbations decreases from $\lambda \approx 2,7.10^{3} \mathrm{~cm}$ at the height $z \approx 400 \mathrm{~km}$ up to $\lambda \approx 6.10^{2} \mathrm{~cm}$ at the heights $z \approx 300 \mathrm{~km}$. While the frequencies $\omega>6.10^{5} \mathrm{~s}^{-1}$ can be easily detected by the MARSIS radar on board the Mars Express the corresponding wavelengths are too small for a 40-m antenna. Nevertheless, we expect that nonlinear processes can change the spectrum significantly, thus allowing the registration of the waves by the MARSIS radar.

The details of the pitch angle distribution function of photelectrons can give important information about the interaction of the electrons with these waves. Our estimates show that the resonance electrons have energies $W_{r} \approx 5-6 \mathrm{eV}$. Due to the interaction of these low energy electrons with the excited waves, the distribution of photoelectrons along the magnetic field line should become more or less flat within this energy range, to diminish the growth rate of the instability. This is in contrast to the observed source-cone distribution at larger energies. So it would be interesting to measure the pitch angle distribution of photoelectrons at such small energies. Unfortunately, due to positive charging of MGS, only electrons with the energies $W>10 \mathrm{eV}$ were detected by Liemohn et al. (2003). Observations of $>3 \mathrm{eV}$ electron fluxes were made on board the Phobos-2 spacecraft, but the detailed pitch angle distributions were not measured (Verigin et al., 1991).

We have also discussed the excitation of electrostatic waves with the frequencies of the order of the electron gyrofrequency. We expect these waves to be generated, first of all, near the terminator, where the loss-cone ditribution of thermal electrons can be formed. While we concentrated on the waves with the frequencies below the electron gyrofrequency $\omega<\omega_{H e}$, waves with higher frequencies $\omega>\omega_{H e}$ can also be excited. But the maximum growth rate of the instability is achieved for the lowest frequency range, $\omega<\omega_{H e}$, that corresponds to the electrostatic whistlers. Such electrostatic whistlers in the presence of plasma irregularities transform into ordinary whistler mode waves that can escape far away from the region of generation.

At the present time there are two indications that plasma waves are excited in the closed magnetic loop regions. First, according to Krymskii et al. (2003), the electrons inside such loops are hotter than outside. It can be caused, at least partly, by the interaction with waves. Second, the peculiar nearisotropic pitch angle distribution of the high energy photoelectrons $(W>100 \mathrm{eV})$ can be explained by the interaction and scattering of electrons by whistler mode waves (Liemohn et al., 2003).

In our paper we have analysed only two types of instabilities which might be excited in particular regions of the ionosphere of Mars. We didn't discuss physical processes in the vicinity of the cusp-type magnetic configurations or close to the magnetic pile-up boundary where the acceleration of electrons can take place. It is known that fast electrons can be the source of wave excitation. We have not investigated low frequency gradient-drift instabilities caused by inhomogeneities of plasma distribution and magnetic fields. Such instabilities play a significant role in the Earth's ionosphere and can also be expected in the ionosphere of Mars.

Acknowledgements. This research was funded by "Bundesministerium für Bildung and Forschung" through "Deutsche Zentrum für Luft- und Raumfahrt e.V.(DLR) Grant \#50 QM 0004.

Topical Editor M. Lester thanks R. Pfaff for his help in evaluating this paper.

\section{References}

Acuña, M. H., Connerney, J. E. P., Wasilevsky P., Lin, R. P., Anderson, K. A., Carlson, C. W., and McFadden, J.: Magnetic fields and plasma observations on Mars: Initial results on Mars Global Surveyer mission, Science, 279, 1676-1680, 1998.

Bespalov, P. A. and Trakhtengerts, V. Yu.: Cyclotron instability of the Earth's radiation belts. In: Reviews of Plasma Physics, 10, Consultants Bureau, New York, 155-284, 1986.

Erokhin, N. S. and Moiseev, S. S.: Wave processes in an inhomogeneous plasma. In: Reviews of Plasma Physics, 7, Consultants Bureau, New York, 181-251, 1979.

Gurnett, D. A.: The Earth as a radio source. Terrestrial kilometric radiation, J. Geophys. Res., 79, 4277-4238, 1974. 
Karpman, V. I, Alekhin, Yu. K., Borisov, N. D., and Rjabova, N. A.: Electrostatic waves with frequencies exceeding the gyrofrequency in the magnetosphere, Astrophys. Space Sci., 22, 267278, 1973.

Karpman, V. I., Alekhin, Yu. K., Borisov, N. D., and Rjabova, N. A.: Electrostatic electron-cyclotron waves in plasma with loss-cone distribution, Plasma Phys., 17, 361-372, 1975.

Kennel, G. F., Scarf, F. L., Frederics, R. W., McGehee, J. H, and Coronity, F. V.: VLF electric field observations in the magnetosphere: J. Geophys. Res., 75, 6136-6152, 1970.

Krasnopolsky., V. A.: Mars' global atmosphere and ionosphere at low, medium and high solar activities: applications for evolution of water, J. Geophys. Res., 107, 5128, doi:10.1029/2001JE001809, 2002.

Krymskii, A. M., Breus, T. K., Ness, N. F., Hinson, D. P., and Bojkov, D. I.: Effect of crustal magnetic field on the near terminator ionosphere at Mars: Comparison of in situ magnetic field measurements with the data of radio science experiment on board Mars Global Surveyor, J. Geophys. Res., 108, 1431, doi:10.1029/2002JA009662, 2003.

Lejeune, G. and Wormser, F.: Diffusion of photelectrons along a field line inside the plasmasphere, J. Geophys. Res., 81, 29002916, 1976.

Liemohn, M. W., Mitchell, D. L., Nagy, A. F., Fox, J. L., Reimer, T. M., and Ma. Y.: Comparison of electron fluxes measured in the crustal fields at Mars by the MGS magnetometer/electron reflectometer instrument with a B field-dependent transport code, J. Geophys. Res., 108(E12), 5314, doi:10.1029/2003JE002158, 2003.
Mikhailovskii, A. B.: Theory of plasma instabilities. Consultants Bureau, New York, 1974.

Mitchell, D. L., Lin, R. P., Mazelle, C., Rème, H, Cloutier, P. A., Connerney, J. E. P., Acuña, M. H., and Ness, N. F.: Probing Mars' crustal magnetic field and ionosphere with MGS electron reflectometer, J. Geophys. Res., 106, 23 419-23 427, 2001.

Nielsen, E.: Mars Express and Marsis, Space Sci. Rev., 111, 245262, 2004.

Pastukhov, V. P.: Classical longitudinal plasma losses from open adiabatic traps, in: Reviews of Plasma Physics, v. 13, Consultants Bureau, New York, 203-259, 1987.

Shinagawa, H.: The ionospheres of Venus and Mars, Adv. Space Res., 33, 1924-1931, 2004.

Treumann, R. A.: Planetary radio emission mechanisms: A Tutorial, Radio Astronomy at Long Wavelengths, in: Geophysical Monograph, 119, American Geophisycal Union, 13-26, 2000.

Verigin, M. I., Gringanz, K. I., Shutte, N. M., Haider, S. A., Szego, K., Kiraly, P., Nagy, A. G., and Gombosi, T. I.: On the possible source of the ionization in the nighttime Martian ionosphere, 1. Phobos 2 HARP electron spectrometer measurements, J. Geophys. Res. 96, 19307-19313, 1991. 